\title{
Perioperative Use of Systemic Lidocaine
}

\section{TO THE EDITOR:}

We read with great interest Terkawi's (1) study that analyzes the incidence of chronic postsurgical pain (CPSP) after mastectomy between patients receiving intravenous (IV) lidocaine versus placebo.

They concluded that perioperative lidocaine administration was associated with a decreased incidence of CPSP, suggesting a protective effect of lidocaine in these patients.

Patients were randomized to receive either an IV lidocaine infusion ( $2 \mathrm{mg} / \mathrm{kg} / \mathrm{h}$ lidocaine), or placebo (normal saline infusion). However, all patients received a bolus of $1.5 \mathrm{mg} / \mathrm{kg}$ of lidocaine prior to induction of anesthesia. The justification for this bolus in the placebo group was not made and did not appear to mirror the otherwise very similar protocol used by Grigoras et al (2), where there was a saline bolus given in place of this study's lidocaine bolus.

The benefits of systemic administration of lidocaine include peripheral and central analgesia by blocking sodium and potassium channels andN-MethylD-aspartate receptors, reduction of neutrophil accumulationand the release of inflammatory mediators and suppression of $C$ fiber evoked potential and secondary hyperalgesia (3-6). The antinociceptive effect of IV lidocaine has been shown to persist after the plasma level has decreased, being more pronounced when administered preoperatively (6). So can a group receiving a bolus of lidocaine be called a "placebo group?" It seems conceivable that a single bolus of lidocaine could be enough to decrease pain without being followed by infusion. This could be the cause of insignificant statistical differences in postoperative pain, opioid consumption, and duration of hospitalization between groups found by the authors in their previous publication (4), but more importantly for this study, may have reduced the magnitude of the effect seen here. Given that this is a secondary analysis, which appears to be underpowered for CPSP as a primary outcome, anything that potentially muddies the water would seem imprudent.

Studies do support this idea. A single bolus of lidocaine at $1.5 \mathrm{mg} / \mathrm{kg}$ has been shown to reduce the incidence and severity of pain for up to 6 hours postoperatively (7), as well as increase propofol potency (8) and reduce the minimum alveolar concentration of sevoflurane (9). Protocols for the use of lidocaine in chronic pain are widely varied (3), and as such it is difficult to make a judgement on the protocol used here. CPSP is a common complication after breast cancer surgery with a prevalence as high as 52\% (10). Perioperative IV lidocaine is a promising drug that has been shown to be useful in treating different types of chronic and postoperative pain $(3,5,7)$. However, to be able to reach acceptable conclusions, well defined protocols and standardization are required.

\author{
Maria Paz Sebastian, MBChB \\ University College London Hospital \\ London, UK \\ E-mail: sebastianmariapaz@gmail.com
}

Simeon West, MBChB

University College London Hospital

London, UK

E-mail: simeon.west@nhs.net 


\section{References}

1. Terkawi AS, Sharma S, Durieux ME, Thammishetti S, Brenin D, Tiouririne $M$. Perioperative lidocaine infusion reduces the incidence of post-mastectomy chronic pain: A double-blind, placebocontrolled randomized trial. Pain Physician 2015; 18: E139-146.

2. Grigoras A, Lee P, Sattar F, and Shorten G. Perioperative intravenous lidocaine decreases the incidence of persistent pain after breast surgery. Clin J Pain 2012; 28:567-572.

3. Kosharskyy B, Almonte W, Shaparin N, Pappagallo M, Smith H. Intravenous infusions in chronic pain management. Pain Physician 2013; 16:231-249.

4. Terkawi AS, Durieux ME, Gottschalk A, Brenin D, Tiouririne M. Effect of intravenous lidocaine on postoperative recov- ery of patients undergoing mastectomy: A double-blind, placebo-controlled randomized trial. Reg Anesth Pain Med 2014; 39:472-477.

5. Marret E, Rolin M, Beaussier M, Bonnet F. Meta-analysis of intravenous lidocaine and postoperative recovery after abdominal surgery Br] Surg 2008; 95:1331-1338.

6. Cursino-Menezes T, Cavalcanti- Lima L, Menezes-Couceiro L, Moraes-Valença M. Intravenous lidocaine to treat postoperative pain. Rev Dor 2014; 15:55-60.

7. Xu YJ, Wang SL, Ren Y, Zhu Y, Tan ZM. A smaller endotracheal tube combined with intravenous lidocaine decreases post-operative sore throat - a randomized controlled trial. Acta Anaesthesiol Scand 2012; 56:1314-1320.

8. Weber U, Krammel M, Linke S, Hamp
T, Stimpfl T, Reiter B, Plöchl W. Intravenous lidocaine increases the depth of anaesthesia of propofol for skin incision - a randomised controlled trial. Acta Anaesthesiol Scand 2015; 59:310-318.

9.- Hamp T, Krammel M, Weber U, Schmid R, Graf A, Plöchl W. The effect of a bolus dose of intravenous lidocaine on the minimum alveolar concentration of sevoflurane: A prospective, randomized, double-blinded, placebo-controlled trial. Anesth Analg 2013; 117:323-328.

10. Alves Nogueira Fabro E, Bergmann A, do Amaral E Silva B, Padula Ribeiro AC, de Souza Abrahão K, da Costa Leite Ferreira MG, de Almeida Dias R, Santos Thuler LC. Post-mastectomy pain syndrome: incidence and risks. Breast 2012; 21:321-325.

\section{In Response to Perioperative Use of Systemic Lidocaine}

We would like to thank Dr. Sebastian and Dr. West for their insightful remarks regarding our recent publications on the effect of intravenous lidocaine on postsurgical acute (1) and chronic pain (2) following breast cancer surgery.

A single bolus of lidocaine is highly unlikely to make any long-term difference. Laboratory data show that a prolonged exposure is necessary to influence receptor functioning and neutrophil priming (3). A previous clinical trial showed significant beneficial effects of intravenous lidocaine infusion on postoperative opioid consumption, despite the fact that control patients also received an intravenous bolus (4). The study by $\mathrm{Yu}$ et al (5) investigated the pain severity of sore throat but not of surgery. We think sore throat is quite a different issue from surgical pain, and we are not sure one can be extrapolated to the other. The fact that a bolus of lidocaine reduces minimum alveolar concentration and increases propofol potency seems unlikely to be relevant in this setting (i.e., reduce postsurgical acute and chronic pain). Finally, as intravenous lidocaine mitigates the pain of propofol injection (6), lidocaine at induction is the standard of practice in our institution, so it would have been difficult to withhold it from the control group.

\author{
Mohamed Tiouririne, MD \\ Department of Anesthesiology \\ University of Virginia \\ PO Box 800710 \\ Charlottesville, VA \\ E-mail: mt9y@virginia.edu
Abdullah S. Terkawi, MD
Physician Resident
Department of Anesthesiology
University of Virginia
PO Box 800710
Charlottesville, VA
E-mail: asterkawi@gmail.com
Marcel E. Durieux, MD, PhD
Professor of Anesthesiology
University of Virginia
PO Box 800710
Charlottesville, VA
E-mail: durieux@virginia.edu \\ Department of Anesthesiology
}




\section{Reference:}

1. Terkawi AS, Durieux ME, Gottschalk A, Brenin D, Tiouririne M. Effect of intravenous lidocaine on postoperative recovery of patients undergoing mastectomy: a double-blind, placebo-controlled randomized trial. Reg Anesth Pain Med 2014:39: 472-477.

2. Terkawi AS, Sharma S, Durieux ME, Thammishetti S, Brenin D, Tiouririne $M$. Perioperative lidocaine infusion reduces the incidence of post-mastectomy chronic pain: a double-blind, placebo-controlled randomized trial. Pain physician 2015:18: E139-146.
3. Hollmann MW, Herroeder S, Kurz KS, Hoenemann CW, Struemper D, Hahnenkamp K, Durieux ME. Time-dependent inhibition of $G$ protein-coupled receptor signaling by local anesthetics. Anesthesiology 2004:100: 852-860.

4. McKay A, Gottschalk A, Ploppa A, Durieux ME, Groves DS. Systemic lidocaine decreased the perioperative opioid analgesic requirements but failed to reduce discharge time after ambulatory surgery. Anesth Analg 2009:109: 1805-1808.
5. Xu YJ, Wang SL, Ren Y, Zhu Y, Tan ZM. A smaller endotracheal tube combined with intravenous lidocaine decreases post-operative sore throat - a randomized controlled trial. Acta Anaesthesiol Scand 2012:56: 1314-1320.

6. Jalota L, Kalira V, George E, Shi YY, Hornuss C, Radke O, Pace NL, Apfel CC; Perioperative Clinical Research Core. Prevention of pain on injection of propofol: systematic review and meta-analysis. BM] 2011:342: dı110. 